\title{
Radiofrequency ablation of metastatic chondrosarcoma-associated refractory ventricular tachycardia originating from the right ventricular outflow tract: A case report and literature review
}

\author{
XIANGMIN SHI ${ }^{1}$, ZHUO LIANG $^{1}$, JIAN LI $^{1}$, JIANPING GUO $^{1}$, ZHAOLIANG SHAN $^{1}$ and YUTANG WANG $^{2}$ \\ Departments of ${ }^{1}$ Cardiology and ${ }^{2}$ Geriatric Cardiology, The General Hospital of PLA, Beijing 100853, P.R. China
}

Received March 19, 2015; Accepted April 26, 2016

DOI: $10.3892 /$ etm.2016.3529

\begin{abstract}
Ventricular tachycardia (VT) and premature contraction originating from the right ventricular outflow tract (RVOT) usually appear in healthy individuals. Radiofrequency ablation (RFA) is highly effective at resolving this type of arrhythmia. Refractory VT of RVOT is uncommon and occasionally results from cardiac metastasis of extraskeletal mesenchymal chondrosarcomas (ESMC). ESMC is a rare malignant tumor arising from soft tissues. The current study presents the case of a 25-year-old male with severe VT arising from RVOT due to metastasis of an ESMC that originally occurred in the retroperitoneum. The diagnosis was confirmed following echocardiography and cardiac magnetic resonance. VT was eventually eliminated by RFA, and during the 8-month follow-up period the patient did not complain of any palpitations. Holter monitoring confirmed the absence of recurrence.
\end{abstract}

\section{Introduction}

Ventricular arrhythmias of the right ventricular outflow tract (RVOT), including premature ventricular contraction (PVC) and ventricular tachycardia (VT), are usually common in healthy individuals without structural heart disease and appear to be benign with a good prognosis (1). However, previous studies have reported that malignant arrhythmias, such as spontaneous ventricular fibrillation (VF) and polymorphic ventricular tachycardia, are occasionally initiated by ventricular extrasystoles arising from RVOT (2,3). Generally, the majority of PVCs and VTs originating from RVOT can be effectively eliminated by catheter radiofrequency ablation (RFA) (4) targeting the initiating ventricular extrasystole. It is thought that the majority of RFA failures can be attributed to

Correspondence to: Dr Yutang Wang, Department of Geriatric Cardiology, The General Hospital of PLA, 28 Fu Xing Road, Beijing 100853, P.R. China

E-mail:wyt301@sina.com

Key words: ventricular tachycardia, right ventricular outflow tract, mesenchymal chondrosarcomas, radiofrequency ablation, cardiac metastasis inappropriate mapping and location of the target (5). Cardiac metastatic tumors are uncommon and usually appear in the pericardium (6). Malignant tumor-associated myocardial infiltration is even more rarely observed in clinical practice and may potentially form arrhythmogenic foci (6). Presented herein is the case of a 25-year-old male patient who suffered refractory monomophic VT of RVOT origin and underwent two unsuccessful RFAs under the guidance of a CARTO system prior to hospitalization. A series of examinations, including echocardiography, chest computed tomography (CT), cardiac magnetic resonance and intracardiac electrophysiological study (EPS), revealed that cardiac metastatic extraskeletal mesenchymal chondrosarcomas (ESMCs) of RVOT origin gave rise to VT, which was resistant to many antiarrhythmic drugs (AAD), and was eventually resolved by RFA therapy.

\section{Case report}

A 25-year-old male farmer who did not present any structural heart disease was admitted to the General Hospital of PLA (Beijing, China) in April 2014 following recurrent palpitations and shortness of breath for 16 months. On admission, no abnormalities were detected following a routine 12-lead surface electrocardiography (ECG; Fig. 1A). Holter monitoring indicated frequent PVC and recurrent monomophic VT (Fig. 1B), which originated from RVOT as determined by a traditional VT localization algorithm (7). However, although the patient underwent two rounds of RFA localized to the RVOT using a three-dimensional mapping system (CARTO) in the Henan Province Hospital, ventricular arrhythmia reoccurred 3 and 6 days after each procedure, respectively. Two weeks prior to hospitalization at the General Hospital of PLA in April 2014, the number of VT episodes markedly increased and occasionally the VT of the patient spontaneously accelerated to $>200$ beats $/ \mathrm{min}$. In addition, VT degenerated into a sustainable pattern resistant to many AADs, which resulted in hemodynamic instability and severely impacted the quality of life of the patient. The ECG morphology of the PVC and VT showed a simple positive R wave in lead II, III, AVF and QS in lead V1, as well as a positive $\mathrm{R}$ wave in lead V6 with $\mathrm{R} / \mathrm{S}$ transition zone (first precordial lead with $\mathrm{R} / \mathrm{S}$ ratio $>1$ ) in lead V3, which was similar to the ECG morphology of the left bundle branch block. These ECG characteristics fulfilled the diagnostic criteria of idiopathic VT of RVOT origin, however, 
compared with idiopathic VT manifesting constant ECG morphology, ambulatory ECG records indicated intermittent small morphological changes of VT in many leads. A notched $\mathrm{R}$ wave in lead III and AVF, and a higher $\mathrm{R}$ wave in lead I intermittently appeared. Simultaneously, an R/S transition zone in precordial leads fluctuated between V3 and V4.. Holter monitoring indicated that the ventricular rates of VT were markedly altered at 90-210 beats/min (Fig. 1B). Physical examination and the results of the laboratory tests carried out using Cobas 6000 and Modular P800 analyzers (Roche Diagnositics, Inc., Rotkreuz, Switzerland), including $0.3 \mathrm{mg} / \mathrm{dl} \mathrm{C}$-reactive protein (normal range, $0-0.8 \mathrm{mg} / \mathrm{dl}$ ), $2 \mathrm{~mm} / \mathrm{h}$ erythrocyte sedimentation rate (normal range, $0-20 \mathrm{~mm} / \mathrm{h}$ ), cardiac-specific enzymes including $0.008 \mathrm{ng} / \mathrm{ml} \mathrm{TNT} \mathrm{(normal} \mathrm{range,} 0-0.1 \mathrm{ng} / \mathrm{ml}$ ) and CK-MB $0.67 \mathrm{ng} / \mathrm{ml}$ (normal range, 0-6.5 ng/ml ), $0.3 \mathrm{mg} / \mathrm{l}$ D-dimmer (normal range, $<0.5 \mathrm{mg} / \mathrm{l}$ ), $143.8 \mathrm{ng} / \mathrm{ml}$ Pro-B-type natriuretic peptide (normal range, $<150 \mathrm{ng} / \mathrm{ml}$ ), serum electrolytes including $4.1 \mathrm{mmol} / 1$ potassium (normal range, 3.5-5.5 mmol/1), $138 \mathrm{mmol} / 1$ natrium (normal range, $135-145 \mathrm{mmol} / \mathrm{l}$ ) and $0.9 \mathrm{mmol} / \mathrm{l}$ magnesium (normal range, 0.7-1.2 $\mathrm{mmol} / \mathrm{l}$ ) were within normal ranges.

The same patient with VT was previously diagnosed with ESMC in 2012, and underwent tumor surgical resection from the retroperitoneum at the Henan Province Hospital. The patient did not receive chemotherapy or radiotherapy, and did not undergo routine follow-up. Following admission of the patient to the General Hospital of PLA on 14th April, 2014, an enhanced chest CT scan revealed extensive nodular mass infiltration in the pleura, pericardium, lung and mediastinum of the patient (Fig. 3A). These results suggested the tumor had metastasized and involved the cardiovascular system. Subsequent trans-thoracic echocardiograghy indicated the presence of a tissue mass attached to the anterior free wall of the RVOT (Fig. 3B and C). These results were further confirmed by cardiac magnetic resonance (CMR), which detected an irregular oval mass infiltrated in the myocardium of the RVOT and protruding into the right ventricular chamber (Fig. 4A and B). Based on these clinical findings, it was suggested that the refractory ventricular arrhythmia of the patient may be closely associated with or may directly result from a metastatic tumor within the RVOT. However, due to the patient's frequent episodes of VT, surgical resection of the tumor, chemical therapy and radiation therapy were not recommended as a treatment strategy. Due to the relatively stable general condition of the patient who had a likely life expectancy of $>1$ year, and due to the fact that sustained VT could further impair heart function and degenerate into malignant life-threatening arrhythmia, a third RFA procedure was attempted with the aim to control ventricular arrhythmia.

The present study was approved by the ethical committee of the general hospital of PLA. The patient provided informed consent and underwent an electrophysiological study following fasting and under sedation; amiodarone (Sanofi, Gentilly, France) and metoprolol (AstraZeneca, London, UK), which had been taken by the patient for 6 months prior to admission to the General Hospital of PLA, were not discontinued with the purpose of reducing the heart rate in the presence of VT. Intracardiac electrograms were recorded using an electrophysiology system (CardioLab; GE Healthcare Bio-Sciences, Pittsburgh, PA, USA). A decapolar mapping catheter (Biosense
Webster, Inc., Diamond Bar, CA, USA) was positioned in the apex of the right ventricle, and a $3.5 \mathrm{~mm}$ saline-irrigated catheter (NAVISTAR ThermoCool ${ }^{\circledR}$; Biosense Webster, Inc.) was positioned into the RVOT via the right femoral vein using a standard Brockenbrough technique (1). The mapping and RFA procedures were performed under the guidance of a CARTO system (Biosense Webster, Inc.). A heparinized saline solution was continuously infused through the sheath ( $2 \mathrm{ml} / \mathrm{min}$ ) to avoid formation of thrombi or air emboli. Systemic point-by-point activation mapping was applied in the presence of VT, the earliest bipolar activity and recording of a local unipolar QS pattern (Fig. 5C) were used to identify the target. The earliest local ventricular activation recorded from the distal electrode pair of the ablation catheter should precede the onset of the surface QRS complex by $15-45 \mathrm{~ms}$. As the RVOT was infiltrated with tumors, and VT originated from numerous sites within the RVOT, the earliest activation site changed according to the various VT origins, ranging from the anterior ventricular septum to the anterior free wall of the RVOT (Fig. 5A and B). An RF current was delivered to a target point at a target temperature of $45^{\circ} \mathrm{C}$, a maximum power of $35 \mathrm{~W}$ and an infusion rate of $17 \mathrm{ml} / \mathrm{min}$ (Stocker Generator; Biosense Webster Inc.). The target point met the following criteria: The local activation of the distal electrode pair of the ablation catheter preceded the onset of surface ECG QRS by $\geq 15 \mathrm{~ms}$; acceleration of the tachycardia followed by gradual slowing during ablation were observed; abrupt termination occurred during ablation; rapid intrinsic deflection in the unipolar QS pattern was observed.

It was revealed that the majority of successful targets were located between the anterior ventricular septum and the free wall, which was compatible with the mapping results. As VT originated from various adjacent sites, a target point with the earliest activation was successfully eliminated during the process of RFA, and another burst of VT with various rates and morphologies appeared a few seconds later, which was mapped near the previous target points. Following extensive RFA in the area of interest comprising at least 30 target points, the VT episode subsided and gradually disappeared. The end point of the procedure occurred upon the disappearance of spontaneous VT and following non-inducibility of VT by programmed electrical stimulation and/or administration of isoproterenol at the rate of $0.2-0.6 \mu \mathrm{g} / \mathrm{min}$ for $30 \mathrm{~min}$ after ablation.

Following the procedure, the patient no longer complained of palpitations or dyspnea. Ambulatory Holter monitoring revealed one episode of accelerated ventricular rhythm similar to RVOT VT 2 days after ablation. In addition, a CMR scan showed the presence of an enhanced area surrounding the metastatic tumor within the RVOT (Fig. 4C), which was compatible with ablation points. AADs were discontinued 1 month after ablation, and no ventricular arrhythmia was recorded by Holter monitoring in the 8 months follow-up.

\section{Discussion}

Ventricular arrhythmias arising from the RVOT usually occur in the absence of apparent structural heart disease, which is idiopathic and rarely life-threatening, and can be effectively eliminated by RFA with a high success rate (4). The mechanism 
A

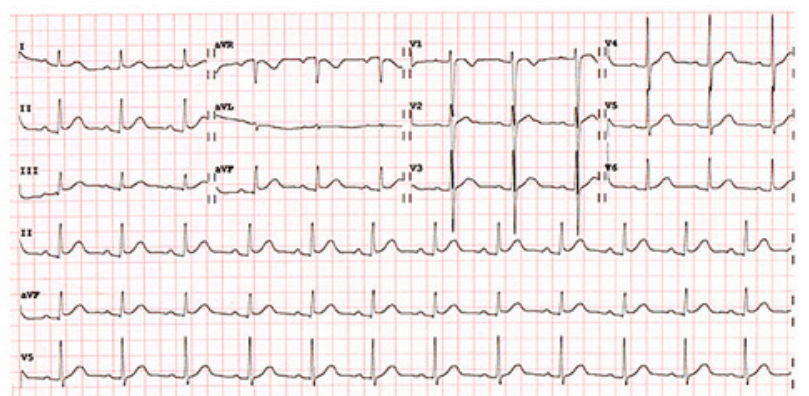

B

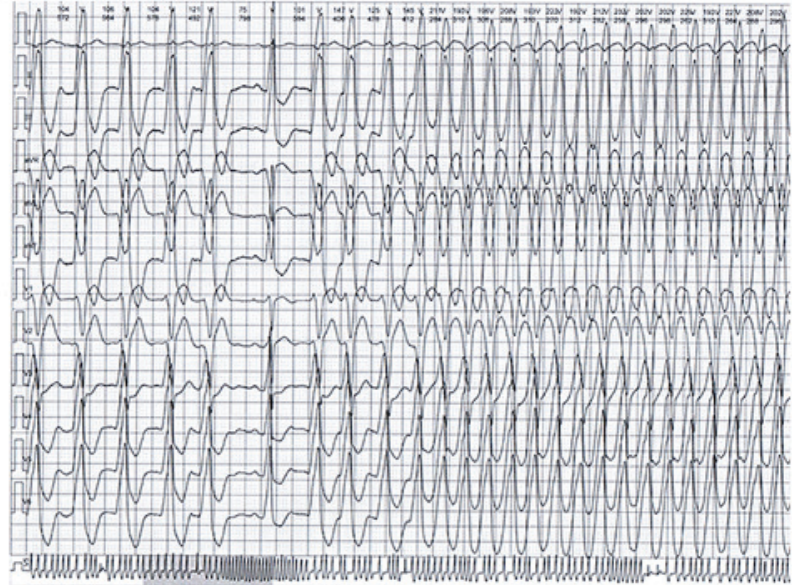

Figure 1. (A) Normal 12-lead electrocardiogram of the patient on admission. (B) Holter recording shows abrupt acceleration of ventricular tachycardia with left bundle branch block and inferior axis.

A

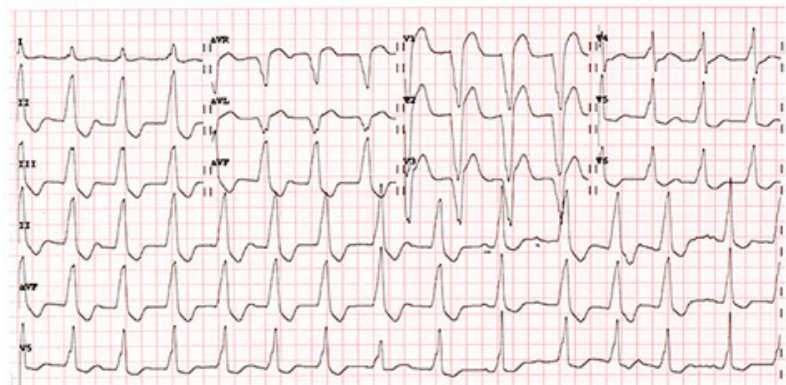

B

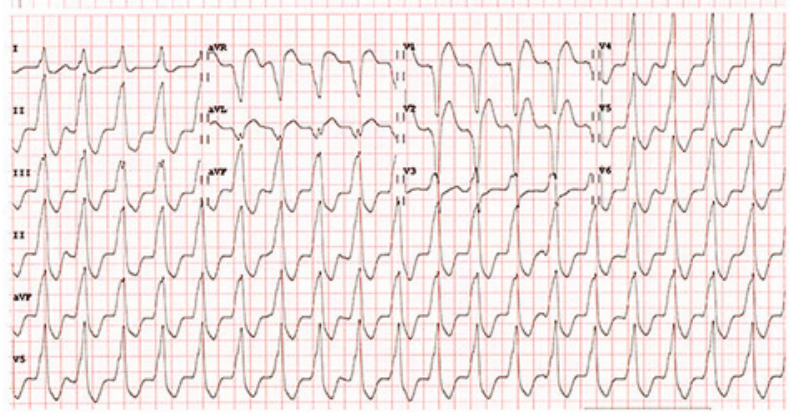

Figure 2. (A) The 12-lead ECG of the VT of the patient shows a precordial $\mathrm{R} / \mathrm{S}$ transition in lead V4 and a peaked $\mathrm{R}$ wave in the lower leads. (B) The 12-lead ECG of the VT of the patient shows a precordial R/S transition in lead V3, a notched $\mathrm{R}$ wave in the lower leads and higher $\mathrm{R}$ waves in lead I ECG, electrocardiogram; VT, ventricular tachycardia.

underlying RVOT VT is thought to be adenosine-sensitive and triggered by cyclic adenosine monophosphate (8). Symptoms vary based on the severity of the arrhythmia and the sensitivity of patients to the rates and duration of VT, and the majority of patients experienced mild to moderate symptoms (9). However,

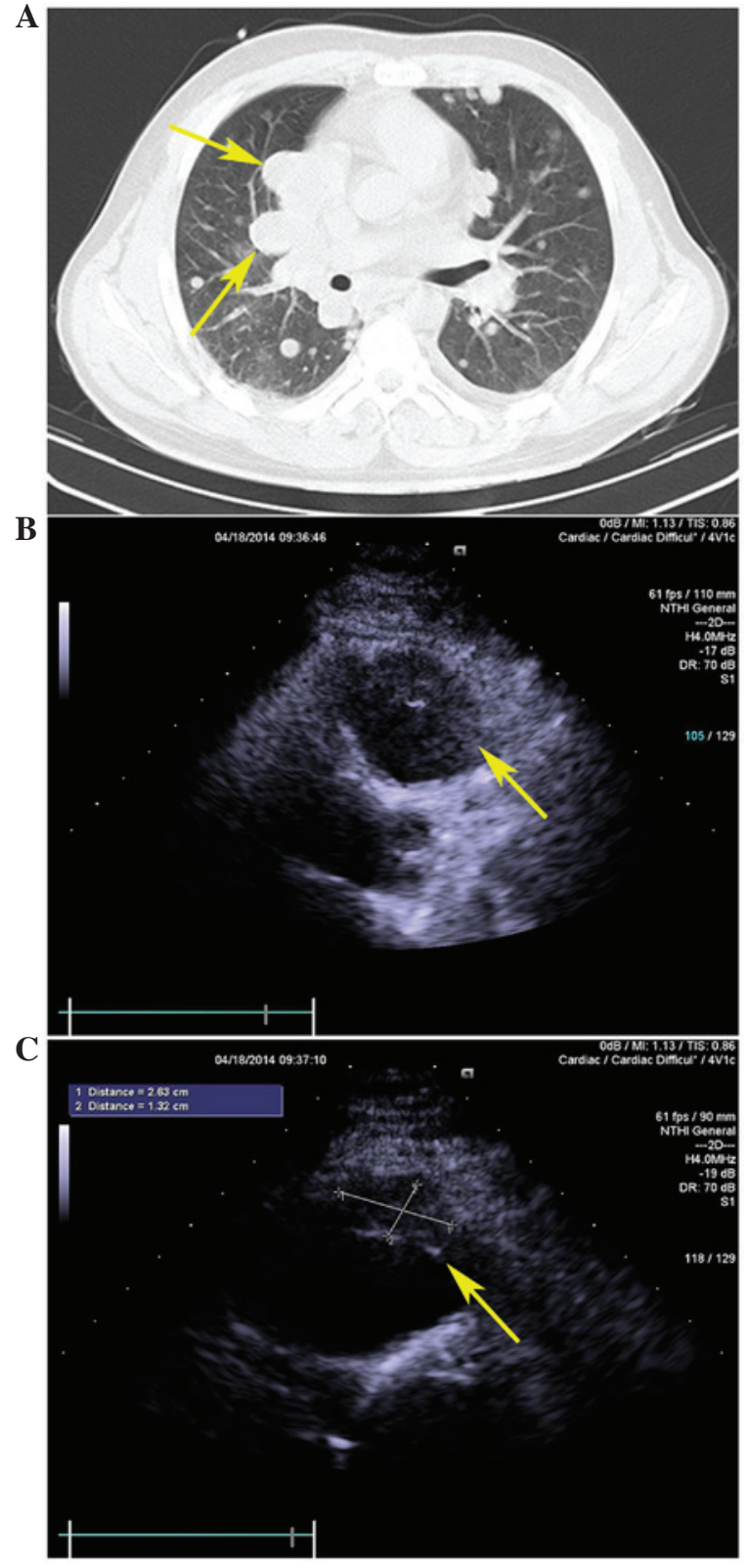

Figure 3. (A) A computed tomography scan of the chest of the patient showed extensive nodular mass infiltration in the lung and mediastinum. (B and C) A transthoracic echocardiogram showed a mass infiltrating the right ventricular outflow tract (indicated by arrows)

in the present study, the patient exhibited self-perpetuating refractory VT leading to severe persistent symptoms, which were markedly different from common idiopathic RVOT VT, and led to the risk of heart failure and sudden death. The patient underwent two rounds of RFA at the Henan Province Hospital; however, VT recurred 3 and 6 days, respectively, after the two procedures. In terms of successful ablation site, the majority of patients with idiopathic VT of RVOT origin have only one site, however, the patient had two different target sites which were not adjacent to each other, which was not compatible with idiopathic VT of RVOT origin. In addition, during the episode of VT, VT manifested various morphologies and rates, which indicated the underlying mechanism may be multiple exits of VT or multiple origins of VT. During the process of EPS, the earliest activation site did not localize to one point, and instead fluctuated between 


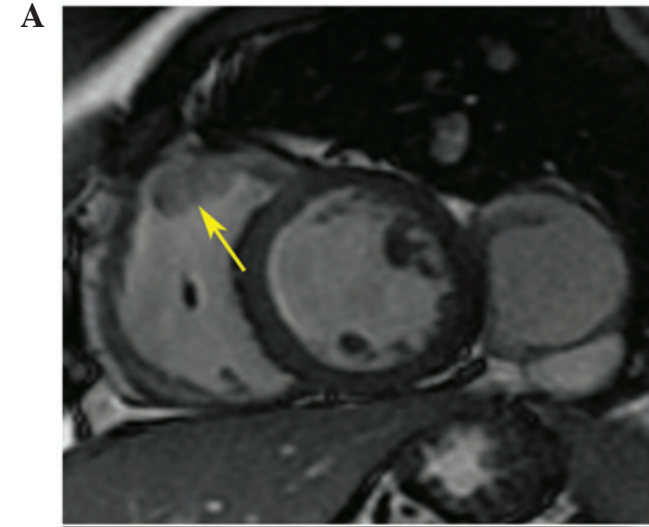

B

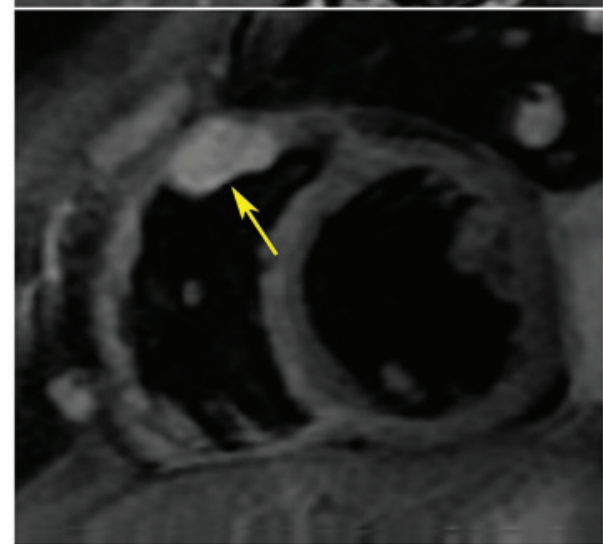

$\mathbf{C}$

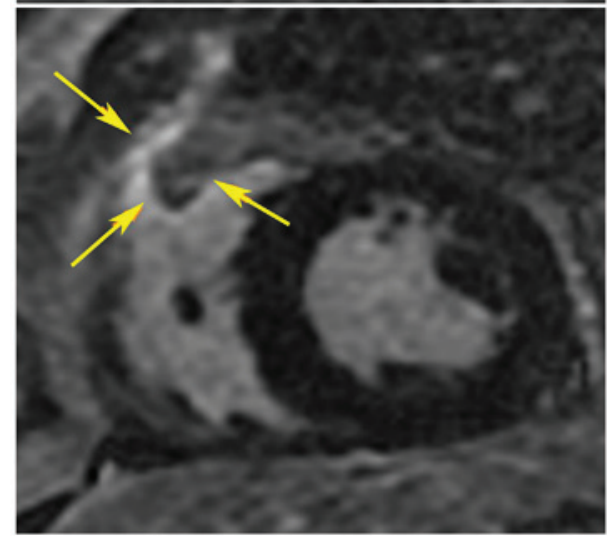

Figure 4. (A) T1-weighted (low signal intensity) cardiac magnetic resonance imaging of the mass (indicated by arrows) in the right ventricular outflow tract. (B) High signal intensity in T2-weighted imaging. (C) High signal intensity post-ablation cardiac magnetic resonance shows the ablation line (indicated by arrows) surrounding the mass.

the free wall and septum within the RVOT, depending on the various morphologies and rates of VT. When VT manifested as a notched $\mathrm{R}$ wave in inferior leads, higher amplitude $\mathrm{R}$ waves in lead I, and early precordial R/S transition zone (V3) with rapid heart rate, the earliest activation point could be recorded near the anterior free wall; however, narrow $\mathrm{R}$ waves in inferior leads with lower amplitude $\mathrm{R}$ waves and later R/S transition zones (V4), as well as a relatively slow heart rate were ECG characteristics associated with the earliest activation site in the anterior septum. These results strongly supported that the VT of the patient may originate from various sites within the RVOT $(10,7)$.

On admission to the General Hospital of PLA, the patient underwent a series of examinations that suggested that a metastatic tumor had infiltrated the RVOT, which may be responsible for the refractory VT and explained the multiple origins of VT. It
A

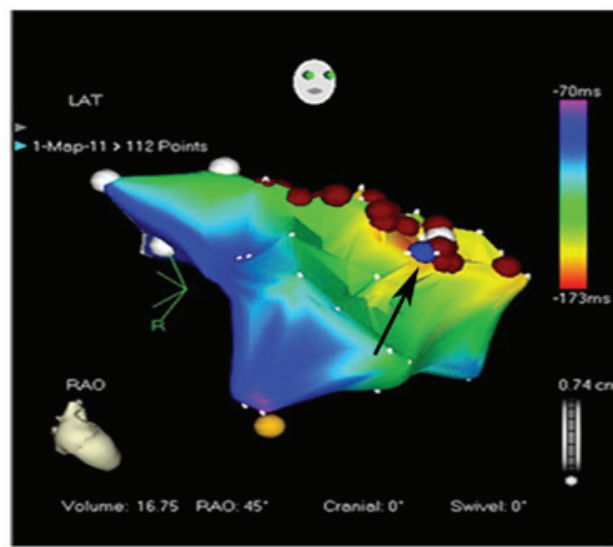

B

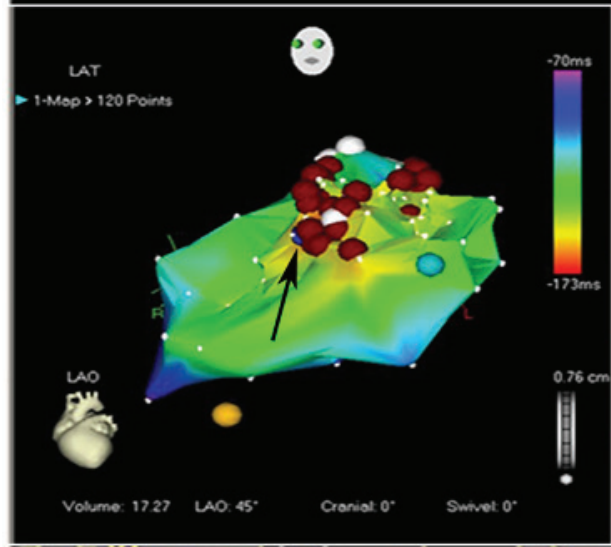

C

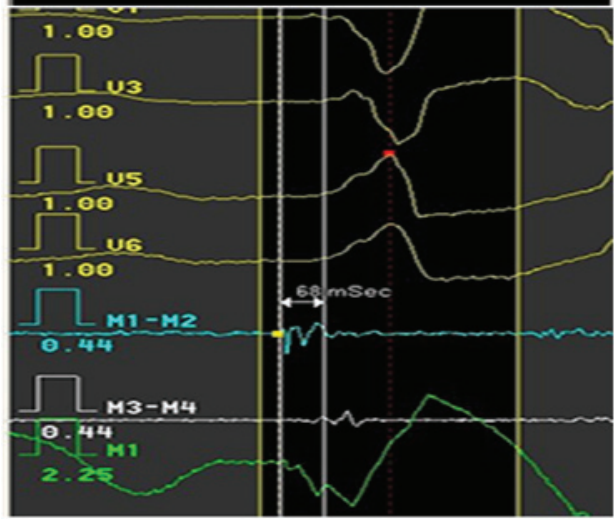

Figure 5. CARTO mapping showing the successful targets (red point) located between the anterior septum and the anterior free wall. (A) right anterior oblique view and (B) left anterior oblique view; the blue point represents one of the successful targets (indicated by a black arrow), (C) in which successful ablation of the VT target included the earliest activation compared with surface ECG. A unipolar QS pattern is a typical feature of successful ablation, which indicates that VT originated from this site.

has previously been reported that cardiac metastatic tumors may result in complete atrioventricular block $(11,12)$ and VT $(13,14)$. Myocardium surrounded or infiltrated by metastatic tumor may manifest abnormal electrophysiological properties (15) and manifested as increased automaticity due to triggered activity, however, severity of myocardial infiltration surrounding the metastatic tumor was not homogeneous and resulted in complicated form of VT with variant ECG morphology and heart rate. The dominant activation pattern was irregular and randomly fluctuated between the anterior free wall and the septum of the RVOT. During VT mapping, if local bipolar activation precedes the QRS of surface ECG by $\geq 15 \mathrm{~ms}$ with a QS pattern of unipolar recording, the site mapped was considered as the ideal 
target for RFA ablation; it was observed that VT accelerated during the initial period of ablation and gradually decelerated or terminated within $30 \mathrm{sec}$, which were typical characteristics of successful target ablation by RF $(16,17)$. After a total of 30 points surrounding the tumor were successfully ablated within the RVOT, VT was not induced by programmed right ventricular stimulation or isoproterenol infusion.

Original cardiac tumors are rarely seen in clinical practice with an incidence rate of $0.02 \%$ (6). However, cardiac metastatic tumors are 20-40 times more frequent than primary cardiac tumors, and predominantly affect the pericardium rather than the myocardium (18). It was reported that $10-12 \%$ cases of malignant tumors were heart-associated at autopsy (18). The patient presented in the current case report was therefore initially underdiagnosed and thought to have idiopathic RVOT-VT; a history of ESMC was not correlated with ventricular arrhythmia. Following hospitalization, a routine chest CT scan indicated the presence of extensive pulmonary metastasis of the primary tumor. It has previously been reported that the heart is the cause of $23 \%$ of systemic metastasis cases (6), which led to the hypothesis that the VT of the patient may result from myocardial infiltration by a tumor. This hypothesis was further confirmed by ECG and CMR scans which outlined the site and extent of the metastatic tumor within the RVOT.

The general condition and past history of patients should be carefully monitored if typical RVOT-VT could not be ablated or recurs following several procedures. In these cases the RVOT may be affected by malignant metastatic tumors and may become an arrhythmogenic foci. CMR is particularly useful for identifying cardiac metastases due to its ability to provide tissue characterization $(19,20)$; the mass-infiltrated RVOT of the patient emitted a low signal intensity on the T1-weighted CMR image and a high signal intensity on the T2-weighted CMR image, which was compatible with the CMR characteristics of malignant tumors and ESMCs $(19,20)$.

Chondrosarcoma is an invasive skeletal tumor with various grades of malignancy, and represents $\sim 11 \%$ of all primary malignant bone tumors (20). This type of tumor rapidly evolves and has a strong tendency to metastasize, and is resistant to chemotherapy (21). Chondrosarcomas usually occur in patients aged 40-80 years, and are more frequent in men (22). $90 \%$ of chondrosarcomas are conventional, and the remaining $10 \%$ include dedifferentiated, clear cell, myxoid and mesenchymal chondrosarcomas (22), with myxoid being the most common subtype. The present case was determined to be a mesenchymal chondrosarcoma as confirmed by pathological examination. ESMC may appear in any location that contains mesenchymal cells; primary sites include limb extremities, the torso, the head and neck, and rarely the heart (23-25). Compared with the myxoid subtype, the ESMC subtype is rare, more aggressive and with poor prognosis due to the high probability of metastases (26), which may occur several years following the initial treatment. Cardiac metastasis of ESMCs is infrequent; it has been reported that the most common site of cardiac metastasis are the right atrium and left intracavity, leading to pulmonary embolism and systemic embolism $(27,28)$. Dyspnea and pleuritic chest pain are the most common symptoms associated with cardiac metastases (29). To our knowledge, RVOT involvement is rarely observed and refractory ventricular arrhythmia has not previously been described as a primary symptom. In the present study, metastatic ESMC-associated VT was effectively treated with RFA rather than surgical resection. During the 8 month follow-up period, the patient did not report any further palpitations, and Holter monitoring confirmed the absence of VT recurrence. Previous studies have reported that the median time from the initiation of cardiac symptoms to the time at which the patient succumbs to the disease was 2 months in the case of cardiac metastases $(30,31)$. However, the general health of the patient reported was relatively stable 8 months following the RFA procedure, and the patient is currently undergoing further treatment for ESMC. The results of the present study suggested that the treatment of VT by RFA targeting following ESMC cardiac metastasis may significantly increase patient lifespan.

\section{References}

1. Calvo N, Jongbloed M and Zeppenfeld K: Radiofrequency catheter ablation of idiopathic right ventricular outflow tract arrhythmias. Indian Pacing Electrophysiol J 13: 14-33, 2013.

2. Kurosaki K, Nogami A, Shirai Y and Kowase S: Positive QRS complex in lead $i$ as a malignant sign in right ventricular outflow tract tachycardia: Comparison between polymorphic and monomorphic ventricular tachycardia. Circ J 77: 968-974, 2013.

3. Shimizu W: Arrhythmias originating from the right ventricular outflow tract: How to distinguish 'malignant' from 'benign'? Heart Rhythm 6: 1507-1511. 2009.

4. Lamba J, Redfearn DP, Michael KA, Simpson CS, Abdollah H and Baranchuk A: Radiofrequency catheter ablation for the treatment of idiopathic premature ventricular contractions originating from the right ventricular outflow tract: A systematic review and meta-analysis. Pacing Clin Electrophysiol 37: 73-78, 2014.

5. Yokokawa M, Good E, Crawford T, Chugh A, Pelosi F Jr, Latchamsetty R, Jongnarangsin K, Ghanbari H, Oral H, Morady F and Bogun F: Reasons for failed ablation for idiopathic right ventricular outflow tract-like ventricular arrhythmias. Heart Rhythm 10: 1101-1108, 2013.

6. Sarjeant JM, Butany J and Cusimano RJ: Cancer of the heart: Epidemiology and management of primary tumors and metastases. Am J Cardovasc Drugs 3: 407-421, 2003.

7. Ito $S$, Tada $H$, Naito $S$, Kurosaki K, Ueda M, Hoshizaki $H$, Miyamori I, Oshima S, Taniguchi K and Nogami A: Development and validation of an ECG algorithm for identifying the optimal ablation site for idiopathic ventricular outflow tract tachycardia. J Cardiovasc Electrophysiol 14: 1280-1286, 2003.

8. Wilber DJ, Baerman J, Olshansky B, Kall J and Kopp D: Adenosine-sensitive ventricular tachycardia. Clinical characteristics and response to catheter ablation. Circulation 87: 126-134, 1993.

9. Nakagawa M, Takahashi N, Nobe S, Ichinose M, Ooie T, Yufu F, Shigematsu S, Hara M, Yonemochi H and Saikawa T: Gender differences in various types of idiopathic ventricular tachycardia. J Cardiovasc Electrophysiol 13: 633-638, 2002.

10. Dixit S, Gerstenfeld EP, Callans DJ and Marchlinski FE: Electrocardiographic patterns of superior right ventricular outflow tract tachycardias: Distinguishing septal and free-wall wits of origin. J Cardiovasc Electrophysiol 14: 1-7, 2003.

11. Mocini D, Longo R, Colivicchi F, Morabito A, Gasparini G and Santini M: A complete atrioventricular block secondary to myocardial metastases of lung cancer. A case report. Ital Heart J 6: 931-932, 2005.

12. Soon CY, Singh D and Ong HY: Myocardial metastatic tumor from a primary oropharyngeal carcinoma presenting as severe 3rd-degree atrioventricular block. Tex Heart Inst J 36: 182-183, 2009.

13. Leak D: Amiodarone for control of recurrent ventricular tachycardia secondary to cardiac metastasis. Tex Heart Inst J 25: 198-200, 1998.

14. Kinoshita K, Hanibuchi M, Kishi M, Kanematsu T, Nishioka Y and Sone S: Case of squamous cell lung cancer with myocardial metastasis complicated with ventricular tachycardia. Nihon Kokyuki Gakkai Zasshi 47: 817-822, 2009 (In Japanese).

15. Miyake CY, Del Nido PJ, Alexander ME, Cecchin F, Berul CI, Triedman JK, Geva T and Walsh EP: Cardiac tumors and associated arrhythmias in pediatric patients, with observations on surgical therapy for ventricular tachycardia. J Am Coll Cardiol 58: 1903-1909, 2011. 
16. Soejima Y, Aonuma K, Iesaka Y and Isobe M: Ventricular unipolar potential in radiofrequency catheter ablation of idiopathic non-reentrant ventricular outflow tachycardia. Jpn Heart J 45: 749-760, 2004.

17. Bogun F, Taj M, Ting M, Kim HM, Reich S, Good E, Jongnarangsin K, Chugh A, Pelosi F, Oral H and Morady F: Spatial resolution of pace mapping of idiopathic ventricular tachycardia/ectopy originating in the right ventricular outflow tract. Heart Rhythm 5: 339-344, 2008.

18. Wada A, Winner M III and Houmsse M: Metastatic melanoma of the right ventricular outflow tract as a cause of ventricular tachycardia. Tex Heart Inst J 41: 103-104, 2014.

19. Sparrow PJ, Kurian JB, Jones TR and Sivananthan MU: MR imaging of cardiac tumors. Radiographics 25: 1255-1276, 2005.

20. Belhocine TZ, Scott AM, Even-Sapir E, Urbain JL and Essner R Role of nuclear medicine in the management of cutaneous malignant melanoma. J Nucl Med 47: 957-967, 2006.

21. Douis H and Saifuddin A: The imaging of cartilaginous bone tumours. II. Chondrosarcoma. Skeletal Radiol 42: 611-626, 2013.

22. Pontes HA, Pontes FS, de Abreu MC, de Carvalho PL, de Brito Kato AM, Fonseca FP, de Freitas Silva BS and Neto NC: Clinicopathological analysis of head and neck chondrosarcoma: Three case reports and literature review. Int J Oral Maxillofac Surg 41: 203-210, 2012.
23. Hsing CT, Oh SY, Lee S, Kwon HC, Kim SH, Park TH, Woo JS, $\mathrm{Na} \mathrm{SH}$ and Kim HJ: Extraskeletal mesenchymal chondrosarcoma of the heart responded to systemic chemotherapy: A case report. Cancer Res Treat 39: 131-133, 2007.

24. Parmar C, Jojo A, Vachhani KC and Vijayan SN: Primary chondrosarcoma of the heart. Eur J Cardiothorac Surg 33: 513-515, 2008.

25. Izzo P, Ricci N, Capolupo R, et al: A rare care of primary chondrosarcoma of the heart. J Cardiovasc Med 8: 210-213, 2007.

26. Hu HJ, Liao MY and Xu LY: Primary retroperitoneal extraskeletal mesenchymal chondrosarcoma involving the vena cava: A case report. Oncol Lett 7: 1970-1974, 2014.

27. Fichaux O, de Muret A, Dessenne X, Rosset P, Pacouret G, Pagot O and Charbonnier B: Cardiac metastasis of chondrosarcoma: A case report. Ann Cardiol Angeiol (Paris) 47: 165-168, 1998 (In French).

28. Oizumi H, Tanaka R, Shimura H, Sasaki K, Koike H, Hattori N and Tanaka S: A case of cerebral embolism with metastatic chondrosarcoma in the left atrium. J Stroke Cerebrovasc Dis 20: 79-81, 2011.

29. Kwon JW, Choi JA, Kwack KS, Oh JH, Chung JH and Kang HS: Myxoid chondrosarcoma in the calcaneus: A case report with MR imaging findings. Skeletal Radiol 36 (Suppl 1): S82-S85, 2007.

30. Leung CY, Cummings RG, Reimer KA and Lowe JE: Chondrosarcoma metastatic to the heart. Ann Thorac Surg 45: 291-295, 1988.

31. Nesi G, Pedemonte E and Gori F: Extraskeletal mesenchymal chondrosarcoma involving the heart: Report of a case. Ital Heart J 1: 435-437, 2000. 